\title{
Estudo quantitativo da lágrima pelo teste de fenol vermelho na população brasileira
}

\author{
Quantitative tear study using the red phenol test in the Brazilian population
}

\author{
Richard Yudi Hida ${ }^{1}$ \\ Maria Cristina Nishiwaki-Dantas ${ }^{2}$ \\ Milton Massato Hida ${ }^{3}$ \\ Kazuo Tsubota ${ }^{4}$
}

\begin{tabular}{|l|}
\hline RESUMO \\
\hline Objetivo: Determinar parâmetros normais do teste de fenol vermelho na \\
população brasileira, e comparar entre diferentes raças, idade e sexo. \\
Métodos: Foram avaliados, como teste de fenol vermelho, 280 indivíduos \\
(560 olhos) da raça branca e 280 indivíduos ( 560 olhos) da raça não branca, \\
que foram divididos de acordo com a idade e o sexo. Foram excluídos \\
indivíduos com qualquer doença ocular, usuários de lente de contato e que \\
usavam medicação ocular. Resultados: Dos 1.120 olhos avaliados, o \\
resultado médio foi de $19,77 \pm 7,90$ mm. Conclusão: O resultado médio \\
encontrado foi um valor intermediárioentre as duas populações previamente \\
estudadas (japonesa e norte-americana).
\end{tabular}

Descritores: Córnea; Fenolsulfonaftaleína/uso diagnóstico; Síndromes do olho seco; Lágrimas; Concentração osmolar; Técnicas de diagnóstico oftalmológico; Comparação transcultural; Estudo comparativo
Trabalho realizado no Departamento de Oftalmologia do Hospital Central da Irmandade da Faculdade de Ciências Médicas da Santa Casa de Misericórdia de São Paulo.

${ }^{1}$ Médico Assistente do Setor de Córnea e Moléstias Externas do Departamento de Oftalmologia da Irmandade Santa Casa de Misericórdia de São Paulo. São Paulo (SP).

${ }^{2}$ Médica Chefe do Setor de Doenças Externas e Coordenadora da Residência Médica do Departamento de Oftalmologia da Irmandade Santa Casa de Misericórdia de São Paulo. São Paulo (SP); Doutora em Medicina pela Universidade Federal de São Paulo (UNIFESP). São Paulo (SP).

${ }^{3}$ Professor Titular aposentado pela Disciplina de Oftalmologia da Universidade Estadual Paulista (UNESP). Botucatu (SP).

${ }^{4}$ Chefe e Professor do Departamento de Oftalmologia da Keio University-School of Medicine. Tokyo, Japão.

Endereço para correspondência: Richard Yudi Hida, Rua Afonso de Freitas, 488 apto. 61 - São Paulo (SP) CEP 04006-052

E-mail: <ryhida@mandic.com.br >

Cada autor declara que não possui interesse financeiro no desenvolvimento ou marketing dos produtos referidos no estudo.

Recebido para publicação em 26.11.2004

Versão revisada recebida em 23.03.2005

Aprovação em 15.04.2005

Nota Editorial: Após concluída a análise do artigo sob sigilo editorial e com a anuência do Dr. Élcio Hideo Sato sobre a divulgação de seu nome como revisor, agradecemos sua participação neste processo.

\section{INTRODUÇÃO}

A ceratoconjuntivite seca provoca um complexo de sintomas causados pela anormalidade da película lacrimal pré-corneal e conjuntival. Vários são os testes clínicos disponíveis para anormalidades do filme lacrimal, porém nenhum pode ser considerado inteiramente eficiente. Entre os testes diagnósticos quantitativos, o mais usado é o teste de Schirmer (I, II e basal). Trata-se de um teste fácil e de baixo custo, porém a colocação das fitas de papel causa desconforto, que pode ser minimizado com a utilização de anestésico tópico (apenas Schirmer basal e II). Após a instilação do colírio, deve-se remover o excesso de líquido que poderia eventualmente umedecer o papel. Além disso, o atrito do papel causa discreta lesão na conjuntiva e na córnea, que inviabiliza a realização imediata do teste de coloração com rosa bengala, pois essas áreas podem sofrer impregnação pelo corante ${ }^{(1-3)}$. Para o teste de Schirmer I, um papel de filtro padronizado (Whatman 41) de $5 \mathrm{~mm}$ de largura e $35 \mathrm{~mm}$ de comprimento, com uma dobra a $5 \mathrm{~mm}$ da extremidade, é colocado no terço lateral da borda palpebral inferior por 5 minutos. Esse método mede a secreção basal e a reflexa da lágrima. No teste de Schirmer com anestesia, instila-se colírio anestésico e o mesmo papel filtro é utilizado. A leitura é feita após 5 minutos. Este método mede a secreção basal da lágrima. O teste de Schirmer II mede o máximo de secreção lacrimal reflexa, através da estimulação da mucosa nasal com uma extremidade de um filamento de algodão. Não há diferença nos valores do teste de Schirmer se os olhos ficarem abertos ou fechados.

O teste do fenol vermelho (RPT ou "Red Phenol Test") (Showa Yakuhin Kako Co., Ltd, Tokyo, Japan \& Menicon USA Inc., Clovis, CA, USA), para quantificar a lágrima, foi descrito por Hamano, com o objetivo de contornar 
as possíveis desvantagens do teste de Schirmer, como variabilidade dos resultados, baixa sensibilidade na detecção de olho seco, desconforto quando realizado sem anestésico tópico e necessidade da instilação prévia de colírio anestésico para medir a secreção basal ${ }^{(4-6)}$. O RPT utiliza um cordão especial de algodão, de aproximadamente $75 \mathrm{~mm}$ de comprimento, banhado em fenol vermelho (fenolsulfonaftaleína), indicador sensível de $\mathrm{pH}$. Sua borda superior é dobrada a $3 \mathrm{~mm}$ da extremidade, para facilitar sua fixação na borda palpebral inferior (Figura 1). Quando em contato com a lágrima, a porção umedecida do cordão passa do amarelo para o vermelho-alaranjado, devido à natureza alcalina da lágrima. A quantidade de lágrima que umedeceu o cordão é medida em milímetros após 15 segundos $^{(4-6)}$.

Estudos demonstram que o teste de fenol vermelho causa pouca sensação de desconforto e pode ser realizado sem anestesia tópica, inclusive com as lentes de contato $^{(6)}$. Apresenta menos variação individual que outros testes e pode ser repetida várias vezes, sem alterar o resultado ${ }^{(6)}$. A pequena dimensão e espessura do fio colocado no fundo de saco conjuntival sugere mínima ou nenhuma injúria à superfície corneal e conjuntival, o que permite a realização da coloração com rosa bengala logo a seguir. Além disso, o teste dura apenas 15 segundos, tempo suficiente para evitar estimulação do lacrimejamento reflexo, enquanto o teste de Schirmer dura 5 minutos ${ }^{(6)}$.

Hamano determinou que a média do RPT para olhos normais no Japão é de $18,8 \mathrm{~mm}^{(7)}$ e Sakamoto constatou que a média do RPT para olhos normais nos Estados Unidos é de

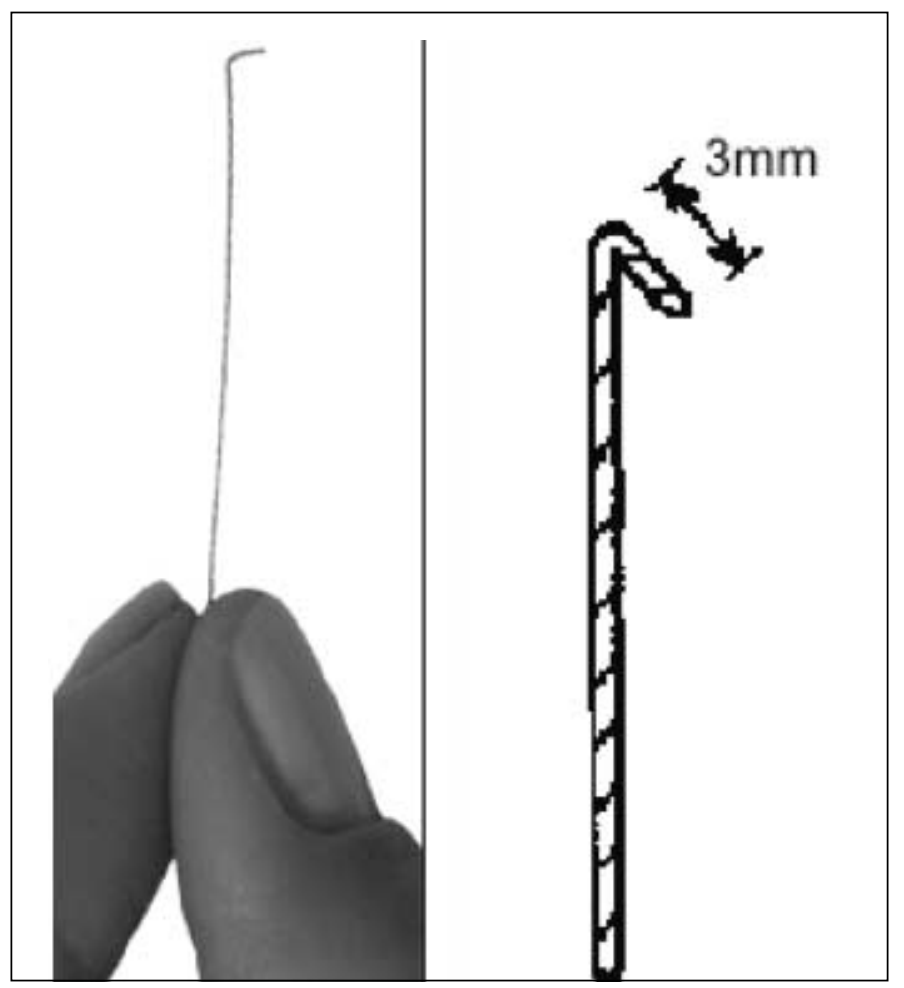

Figura 1 - Aspecto do cordão de fenol vermelho
23,9 mm ${ }^{(6-8)}$. Nas condições do estudo (temperatura e umidade semelhantes para ambas as populações), a diferença entre a população japonesa e americana foi considerada estatisticamente significante, porém não havia nenhum estudo realizado na população miscigenada brasileira.

Este estudo tem como objetivo, analisar e comparar a quantidade de lágrima nas diferentes raças, idade e sexo, pelo teste de fenol vermelho e determinar seus parâmetros normais na população brasileira.

\section{MÉTODOS}

Este estudo foi realizado no Departamento de Oftalmologia da Faculdade de Ciências Médicas da Santa Casa de São Paulo.

Foram estudados com o teste de fenol vermelho, 280 indivíduos (560 olhos) da raça branca e 280 indivíduos (560 olhos) das raças não branca, que foram divididos de acordo com a idade e o sexo (Tabela 1). Foram incluídos os indivíduos que vieram para o nosso serviço para consulta de rotina. Foram excluídos os indivíduos com qualquer doença ocular, usuários de lente de contato e que usavam qualquer medicação ocular.

Foram considerados da raça branca, todos os indivíduos

\begin{tabular}{|lccccc|}
\hline \multicolumn{5}{|c|}{ Tabela 1. Número de olhos examinados divididos por grupo etário, } \\
sexo e raça \\
Idade (anos) & BM & BF & NBM & NBF & Subtotal \\
\cline { 2 - 5 } até 9 & 40 & 40 & 40 & 40 & 160 \\
10 a 19 & 40 & 40 & 40 & 40 & 160 \\
20 a 29 & 40 & 40 & 40 & 40 & 160 \\
30 a 39 & 40 & 40 & 40 & 40 & 160 \\
40 a 49 & 40 & 40 & 40 & 40 & 160 \\
50 a 59 & 40 & 40 & 40 & 40 & 160 \\
> 60 & 40 & 40 & 40 & 40 & 160 \\
Subtotal & 280 & 280 & 280 & 280 & 1120 \\
BM= branco masculino; BF= branco feminino; NBM= não branco masculino; \\
NBF= não branco feminino
\end{tabular}

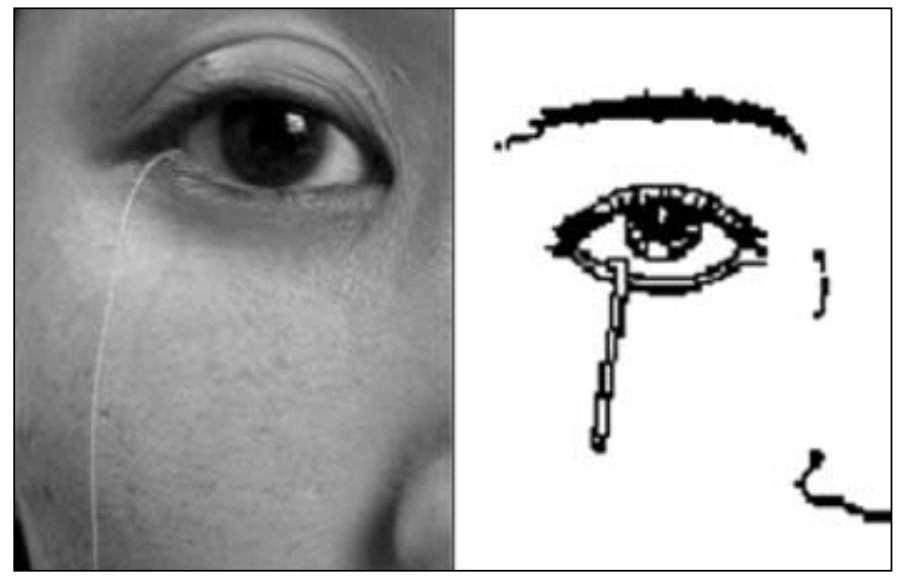

Figura 2 - Aspecto do cordão de fenol vermelho na pálpebra 
com descendência européia de pele branca (sem descendência da raça amarela ou negra). E foram considerados da raça não branca, todos os indivíduos com descendência da raça negra e vermelha (sem descendência da raça amarela).

$\mathrm{O}$ teste foi feito em ambiente fechado, com ausência de vento. A umidade média do ar foi de $65 \%$ e a temperatura média de $23^{\circ} \mathrm{C}$ nas salas dos ambulatórios.

Para evitar variabilidade do resultado, três médicos foram treinados antes da realização do estudo.

Foi preenchido um protocolo para cada paciente em que constava o nome, endereço, profissão, raça, idade e questionário sobre queixas oculares, como sensação de olho seco, olho vermelho, fadiga ocular, lacrimejamento freqüente, fotofobia, incômodo e dor. Foi realizado consentimento verbal em todos os indivíduos.

Os cordões, banhados em fenol vermelho, vêm esterilizados num envelope de alumínio. A porção dobrada era então aberta num ângulo que possibilitasse fácil colocação na conjuntiva palpebral, mais especificamente no terço lateral da pálpebra inferior. Os pacientes eram orientados a manter os olhos abertos em posição primária do olhar e piscando normalmente (Figura 2).

Após 15 segundos, medidos com cronômetro digital, o cordão era retirado delicadamente e depois medida a parte alaranjada em milímetros a partir da ponta do cordão. Foi avaliado um olho de cada vez.

Os resultados foram submetidos a testes estatísticos de Kruskal-Wallis para todas as amostras e Mann-Whitney (comparações múltiplas) para comparar as amostras dos grupos etários e raciais e avaliadas suas significâncias $(\mathrm{p}<0,05)$.

\section{RESULTADOS}

Dos 1.120 olhos avaliados com o teste do fenol vermelho, o resultado médio foi de $19,77 \pm 7,90 \mathrm{~mm}$.

A população masculina branca ( 280 olhos) apresentou

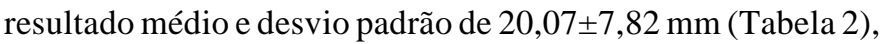
e foi estatisticamente significante $(\mathrm{p}=0,0001)$. No teste de comparações múltiplas, embora os grupos etários mais jovens (de 0 a 9; 10 a 19; e 20 a 29 anos) tenham apresentado quantidade de lágrima maior que os grupos maiores de 60 anos, a diferença não foi estatisticamente significante.
A população feminina branca (280 olhos) apresentou resultado médio de 18,93 $\pm 7,84 \mathrm{~mm}$ (Tabela 2), valor estatisticamente significante $(\mathrm{p}=0,0001)$. No teste de comparações múltiplas, observou-se que a faixa etária de 10 a 19 anos apresentou maior quantidade de lágrima e que os valores foram diminuindo nas faixas etárias mais avançadas, mas os valores não foram estatisticamente significantes.

A população masculina não branca (280 olhos) apresentou resultado médio de $20,75 \pm 7,91 \mathrm{~mm}$ (Tabela 2), valor estatisticamente significante $(\mathrm{p}=0,0063)$. No teste de comparações múltiplas, verificou-se que a faixa etária de 0 a 9 anos, 10 a 19 anos e 20 a 29 anos apresentou maior quantidade de lágrima que os grupos de 30 a 39 anos, 40 a 49 anos e 50 a 59 anos, mas os valores não foram estatisticamente significante.

A população feminina não branca (280 olhos) apresentou resultado médio de 19,34 7 ,95 $\mathrm{mm}$ (Tabela 2), valor também estatisticamente significante $(\mathrm{p}=0,0000)$. No teste de comparações múltiplas, observamos que os grupos etários de 0 a 9 anos, 10 a 19 anos, 20 a 29 anos e 30 a 39 anos apresentaram maior quantidade de lágrima que os grupos de 40 a 49 anos, 50 a 59 anos e maiores de 60 anos, mas os valores não foram estatisticamente significantes.

Comparando-se as quantidades de lágrima entre o sexo feminino e masculino, nos diversos grupos etários, não houve, de modo geral, diferença estatisticamente significante, exceto no grupo masculino não branco, na faixa de 40 a 49 anos, que apresentou maior quantidade de lágrima que as mulheres não brancas na mesma faixa etária.

Não houve diferenças estatisticamente significantes entre as raças branca e não branca.

\section{DISCUSSÃO}

Com este estudo, comparamos o resultado médio do teste do fenol vermelho da população brasileira com os da população japonesa e norte-americana (Tabela 3$)^{(6,8)}$.

Os testes de Schirmer e do tempo de rotura do filme lacrimal têm sido aplicados universalmente, ou seja, os mesmos valores são considerados nas diferentes populações. O estudo comparativo do teste do fenol vermelho, entretanto, demonstrou diferença significante entre os japoneses (apenas de origem asiática) e os norte-americanos brancos, para temperatura e umidade

\begin{tabular}{|c|c|c|c|c|c|c|c|c|}
\hline \multirow[b]{2}{*}{ Raça/sexo } & \multicolumn{7}{|c|}{ Idade (anos) } & \multirow[b]{2}{*}{ Subtotal } \\
\hline & Até 9 & 10 a 19 & 20 a 29 & 30 a 39 & 40 a 49 & 50 a 59 & $>60$ & \\
\hline $\mathrm{BM}$ & $22,45 \pm 7,22$ & $24,88 \pm 5,87$ & $20,98 \pm 8,00$ & $16,43 \pm 8,51$ & $18,65 \pm 7,26$ & $19,90 \pm 6,90$ & $17,23 \pm 7,71$ & $20,07 \pm 7,82$ \\
\hline $\mathrm{BF}$ & $21,63 \pm 6,02$ & $25,33 \pm 7,52$ & $20,78 \pm 7,14$ & $17,70 \pm 6,41$ & $14,90 \pm 5,99$ & $16,13 \pm 5,77$ & $16,03 \pm 7,30$ & $18,93 \pm 7,84$ \\
\hline NBM & $23,78 \pm 8,26$ & $22,75 \pm 6,07$ & $22,70 \pm 6,87$ & $19,58 \pm 8,89$ & $20,78 \pm 8,38$ & $16,70 \pm 6,84$ & $19,00 \pm 7,78$ & $20,75 \pm 7,91$ \\
\hline NBF & $24,23 \pm 7,35$ & $22,48 \pm 6,84$ & $20,30 \pm 7,29$ & $22,45 \pm 7,22$ & $13,75 \pm 6,64$ & $16,50 \pm 9,18$ & $18,93 \pm 5,88$ & $19,34 \pm 7,95$ \\
\hline Subtotal & $23,02 \pm 7,26$ & $23,86 \pm 6,66$ & $21,19 \pm 7,33$ & $18,23 \pm 7,96$ & $17,02 \pm 7,60$ & $17,31 \pm 8,06$ & $17,79 \pm 7,25$ & $19,77 \pm 7,90$ \\
\hline
\end{tabular}




\begin{tabular}{|lc|}
\hline $\begin{array}{c}\text { Tabela 3. Resultado médio do RPT em olhos normais, comparação } \\
\text { entre indivíduos japoneses, norte-americanos e brasileiros }\end{array}$ \\
& X \pm S (mm) \\
Japão* & $18,8 \pm 8,6$ \\
EUA $^{* *}$ & $23,9 \pm 9,5$ \\
Brasil & $19,77 \pm 7,8$ \\
X=média; S= desvio-padrão. \\
Fonte: ${ }^{*}$ Hamano et al. ${ }^{(6) ; ~ *}$ 'Sakamoto et al. ${ }^{(8)}$ \\
\hline
\end{tabular}

ambiental semelhantes. Nosso estudo foi realizado em condições semelhantes de temperatura e umidade e o resultado médio encontrado foi um valor intermediário (mais próximo da japonesa) entre as duas populações previamente estudadas. Sakamoto sugeriu que a diferença anatômica da margem palpebral (menor) ou o menor fundo de saco conjuntival poderiam resultar em um menor menisco lacrimal nos japoneses e uma vez que o teste do fenol vermelho mede basicamente o filme lacrimal residual existente primariamente no fundo de saco conjuntival inferior (com mínimo efeito na secreção reflexa), o menor valor do teste em japoneses estaria explicado ${ }^{(8)}$

Nossa população foi dividida em brancos e não brancos (não foram estudados os de origem oriental), o que não justificaria o valor mais próximo ao dos japoneses, contrariando a teoria de Sakamoto. Poderíamos esclarecer melhor esta variável se comparássemos a população oriental brasileira com nossos achados.

Em geral, sabemos que a produção de lágrima diminui com a idade ${ }^{(2,8)}$, porém em nosso estudo, embora os valores do teste encontrados nas faixas etárias mais avançadas tenham sido menores, a diferença entre as idades não foi considerada estatisticamente significante. Levantamos, assim a hipótese de que talvez a drenagem de lágrima deva diminuir com a idade para manter os níveis de lágrima discretamente diminuídos como os encontrados neste estudo (Gráfico 1).

Assim como em estudos anteriores, os dados não indicam diferença estatisticamente significante dos resultados entre os sexos, exceto no grupo masculino não branco, entre 40 e 49 anos, que apresentou maior quantidade de lágrima que o feminino não branco ${ }^{(8)}$.

Devemos esclarecer que o diagnóstico de olho seco não deve se basear em um único exame. Embora os valores do teste

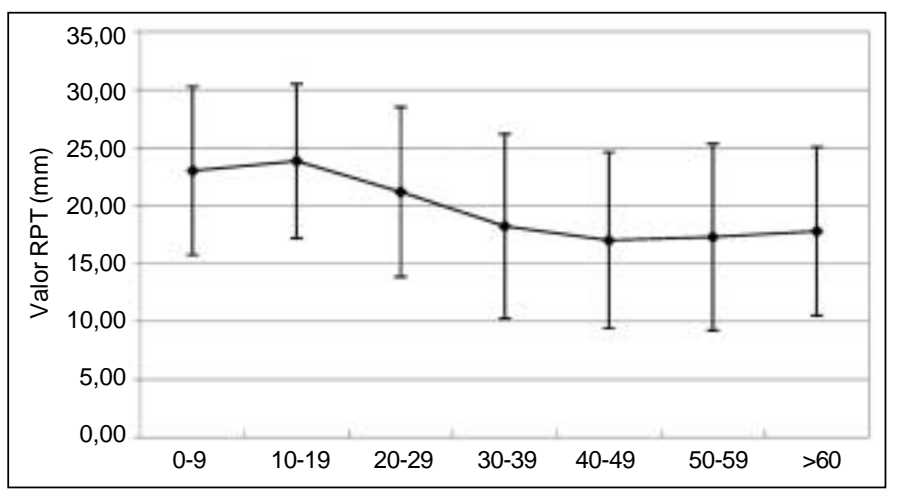

Gráfico 1 - Valores do fenol vermelho (RPT) em milímetros de acordo com os grupos etários da população brasileira, mostrando que os valores diminuem discretamente com a idade de Schirmer e de fenol vermelho não correspondam, o fenol vermelho poderia ser utilizado como alternativa ao Schirmer. Outros testes seriam de grande valor na complementação diagnóstica, como a pesquisa do tempo de rotura do filme lacrimal, a coloração com rosa bengala, o teste de Schirmer II, para avaliação do funcionamento da glândula lacrimal. O diagnóstico é feito da correta interpretação destes exames em conjunto ${ }^{(3)}$.

Alguns autores demonstraram que o teste do fenol vermelho é um teste útil para a análise do filme lacrimal em portadores de olho $\operatorname{seco}^{(9)}$. Uma vez que os nossos resultados em não portadores de olho seco foram semelhantes aos da população americana, também não portadora de olho seco, poderíamos utilizar os valores encontrados nos estudos americanos de olho seco em nossos pacientes com olho seco. Importante salientar que valores abaixo de $10 \mathrm{~mm}$ são considerados anormais ${ }^{(10)}$.

Apesar da controvérsia de seu uso na literatura oftalmológica, estudos recentes revelam que o RPT é considerado uma avaliação quantitativa direta da lágrima no saco conjuntival e mostrou-se sensível para diagnosticar olho $\operatorname{seco}^{(11)}$. Outros autores sugerem que este tipo de teste pode estar associado com a quantificação volumétrica do filme lacrimal ou apenas caracterização de absorção do papel ou do fio ${ }^{(12)}$. A reprodutibilidade e repetitividade destes testes questiona sua validade diagnóstica, mas estudos atuais, mostram relação direta com a validade diagnóstica do corante de fluoresceína, o teste de Schirmer e da meniscometria ${ }^{(12)}$.

\section{CONCLUSÃO}

Subseqüentes estudos estão sendo realizados para correlacionar o teste de RPT com outros testes quantitativos da lágrima na população brasileira.

Com os resultados obtidos e a praticidade do exame, esperamos que este estudo seja útil no uso clínico para investigação de anormalidades do filme lacrimal.

\section{ABSTRACT}

Purpose: To detect normal values of red phenol thread test in the Brazilian population and compare it between different races, age and sex. Methods: 280 white individuals (560 eyes) and 280 non-white individuals (560 eyes) were analyzed regarding sex and age, and analyzed using the Phenol Red test. Individuals with ocular diseases, contact lens or ocular drug users were excluded from this study. Results: Of the 1,120 evaluated eyes, the mean \pm standard deviation result was $19,77 \pm 7,90 \mathrm{~mm}$. Conclusion: The mean result found in this study was an intermediate value compared to the previously studied populations (Japanese and American).

Keywords: Cornea; Phenolsulfonphthalein/diagnostic use; Dry eye syndromes; Tears; Osmolar concentration; Diagnostic techniques, ophthalmological; Cross-cultural comparison; Comparative study 


\section{REFERÊNCIAS}

1. Farris RL, Gilbard JP, Stuchell RN, Mandel ID. Diagnostic tests in keratoconjunctivitis sicca. Clao J. 1983;9(1):23-8.

2. Mendes LE, Santos PM, Parente FSP, Gonçalves JOR, Belfort Jr R. Olho seco em pessoas com idade superior a 40 anos selecionadas em 3 cidades brasileiras. Arq Bras Oftalmol. 1995;62(1):10-5.

3. Nishiwaki-Dantas MC. Olho seco. Arq Bras Oftalmol. 1999;62(1):101-5.

4. Hamano H, Hori M, Mitsunaga S, Kojima S, Maeshima J. Tear test (preliminary report). J Jpn Contact Lens Soc. 1982;24:103-7.

5. Hamano H, Hori M, Hamano T, Mitsunaga S, Maeshima J, Kojima S, et al. A new method for measuring tears. Clao J. 1983;9(3):281-9.

6. Hamano H, Hori M, Maeshima J, Mitsunaga S, Yamotogi K, Kizaki T. Contact lens wear and tear secretion test- fundamental experiment on cotton threads. J Jpn Contact Lens Soc. 1982;24:287-91.
7. Hamano T. The clinical significance of the phenol red thread tear test. Folia Ophthalmol Jpn. 1991;42:719-27.

8. Sakamoto R, Bennett ES, Henry VA, Paragina S, Narumi T, Izumi Y, et al. The phenol red thread tear test: a cross-cultural study. Invest Ophthalmol Vis Sci. 1993;34(13):3510-4

9. Patel S, Farrell J, Blades KJ, Grierson DJ. The value of a phenol red impregnated thread for differentiating between the aqueous and non-aqueous deficient dry eye. Ophthalmic Physiol Opt. 1998;18(6):471-6.

10. Kurihashi K, Yanagihara N, Honda Y. A modified Schirmer test: the fine-thread method for measuring lacrimation. J Pediatr Ophthalmol. 1977;14(6):390-7.

11. Nichols KK, Nichols JJ, Lynn Mitchell G. The relation between tear film tests in patients with dry eye disease. Ophthalmic Physiol Opt. 2003;23(6):553-60.

12. Tomlinson A, Blades KJ, Pearce EI. What does the phenol red thread test actually measure? Optom Vis Sci. 2001;78(3):142-6.

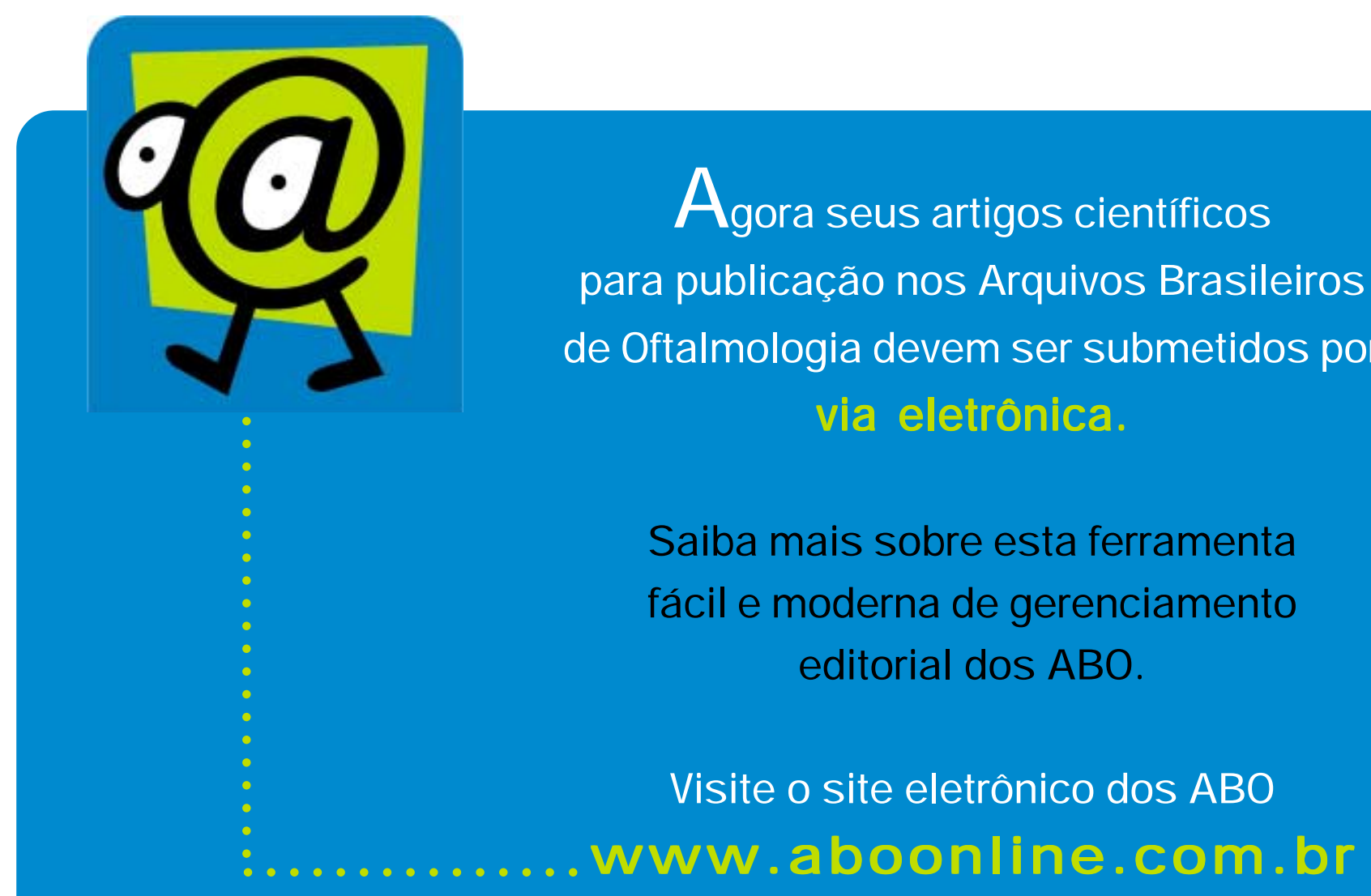

